\title{
ACTINOMYCOSIS: A RARE DISEASE
}

\author{
ABM MONIRUDDIN ${ }^{1}$, HAMIDA BEGUM ${ }^{2}$, KHAIRUN NAHAR $^{2}$, TANVEER AHMED ${ }^{3}$, MALIHA ALAMSIMI ${ }^{4}$, \\ M MAHBUBUR RAHMAN ${ }^{5}$, M KAMRUZZAMAN ${ }^{5}$, MOHAMMADSADIQUL AMIN $^{6}$
}

\begin{abstract}
A 35 year old male presented with multiple discharging sinuses in the right gluteal region \& multiple nodular soft tissue swellings in the right side of face for one year. FNAC from lesions of both sites showed it as a case of actinomycosis. He was successfully treated by antibiotics \& local care of the affected area. No surgical intervention was required. Here we like to present our experience of diagnosing \& treating this rare disease.
\end{abstract}

Keywords: Actinomycosis, Actinomyces israeli, cervicofacial actinomycosis

\section{Introduction}

Actinomycosis, once a common \& fatal disease has declined remarkably owing to advent of potent antimicrobial agents. ${ }^{1}$ Now it has become a more diagnostic challenge as patients no longer commonly present with advanced disease. ${ }^{2}$ Although the causative agents are true bacteria, lesions resemble fungal, mycobacterial \& cancerous ones. First human case was described by Israel in 1878. ${ }^{1,2}$ Actinomycosis is a subacute-to-chronic bacterial infection. The organisms are non-motile, non-spore forming, nonacid fast, Gram positive, pleomorphic, anaerobic-tomicroaerophilic filamentous bacterial rods. ${ }^{3}$ Common causative organisms are ${ }^{1}$ Actinomyces israeli, Actinomyces gerencseriae and other related species. It may involve cervicofacial \& ileocaecal regions, lung, liver, kidney, skin, meninges \& even bone (causing osteomyelitis). ${ }^{4-10}$ The infection is characterized by contiguous spread, granulomas \& formation of multiple abscesses \& sinus tracts discharging 'Sulfur Granules'. Discharging sinus tract or fistula usually becomes secondarily infected with other bacteria. If it is not treated properly it can become complicated \& even endanger life with significant morbidities.

\section{Case Report}

A 35 years old non-diabetic male presented with multiple painless discharging sinuses in the right gluteal region \& multiple nodular soft tissue swellings in the right side of face for one year. He had been well at about one year back when he noticed gradual development of multiple painless nodular lesions at his right gluteal region at the beginning \& later at his face (in the right side) over both lower $\&$ upper jaws. Those at the right gluteal regions soon turned into painless abscesses. These abscesses burst leading to sinus formation. The nodular lesions at the right side of face enlarged slowly to produce disfigurement. He gave no history of oral infection (no dental infection or dental caries or tonsillitis). He had no disturbance in his bowel, bladder \& respiratory functions.

He gave no significant past medical history except an acute abscess in left gluteal region 18 years back which was treated successfully by operation and oral medication. None of his family members \& fellow workers suffered from similar sort of disease.

He was non-alcoholic, but a smoker.

General examination showed nothing contributory other than mild disfigurement (mild wrinkling of skin

1 Consultant, Surgery, General Hospital, Narayanganj.

2 Assistant Professor, Department of Gynaecology \& Obstetrics, BSMMU, Dhaka.

3 Assistant Surgeon, Sadar Upazilla Health Complex, Narayanganj,

4 Medical Officer, Sadar Upazilla Health Complex, Narayanganj

5 Assistant Registrar, Surgery, General Hospital, Narayanganj.

6 Assistant Surgeon, Surgery, General Hospital, Narayanganj.

Correspondence: Dr. ABM Moniruddin, Consultant Surgeon, General Hospital, Narayaganj, E-mail: monirudd@bangla.net 
over the mandible) on the right side of face. Systemic examinations also revealed nothing significant. Examination of the face \& the right gluteal region showed mild disfigurement of face, non-tender freely mobile firm to hard very small nodules of variable sizes on the right side of face, non-tender gluteal sinuses with unremarkable margin (Figures $1 \& 2$ ). The discharges were found clear with occasional yellow to black granules. Local \& regional lymph nodes were not enlarged. Routine blood investigation was normal except neutrophilic leucocytosis, elevated ESR $\left(70 \mathrm{~mm}\right.$ in $\left.1^{\text {st }} \mathrm{hr}\right)$. X-ray of pelvic bones showed no features of osteomyelitis. Ultrasonogram of whole abdomen was normal. Culture of specimen (discharges) in ordinary media showed no growth. FNAC smears from both sites revealed squamous epithelial cells, numerous neutrophils \& some tangled filamentous masses suggestive of filaments of Actinomycosis. No malignant cells or granuloma was found. We started treatment of Actinomycosis by injectable benzyl penicillin and metronidazole for seven days followed by oral phenoxymethyl penicillin, metronidazole coupled with local care (cleaning with normal saline, povidone iodine solution $\&$ application of povidone iodine cream). The response was dramatic. The discharging sinuses healed within months \& the nodular irregularities within the soft tissues over the mandible began to disappear. The patient reported at the end of two months \& four months following the start of the specific treatment for Actinomycosis.

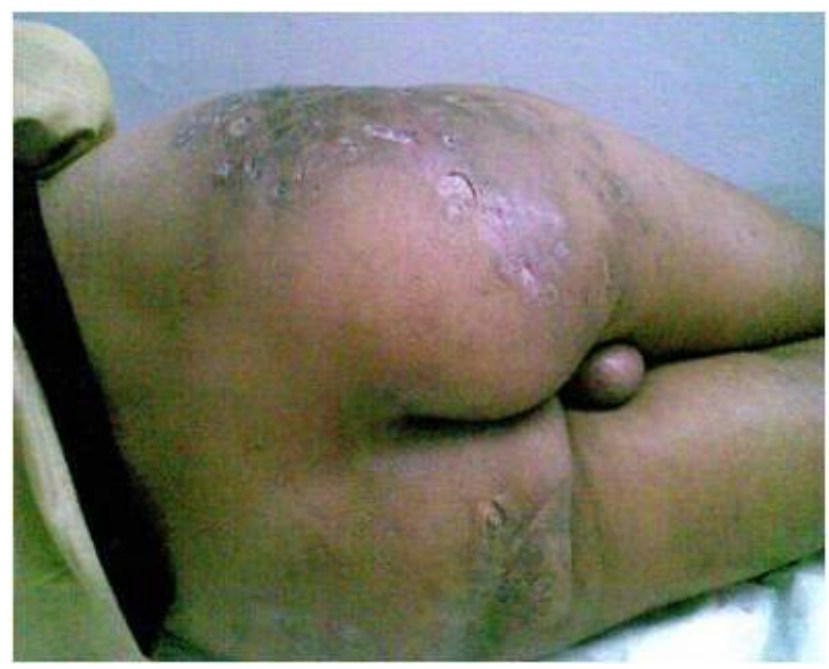

Fig.-1: Healed gluteal lesions (four months after initiation of specific treatment).

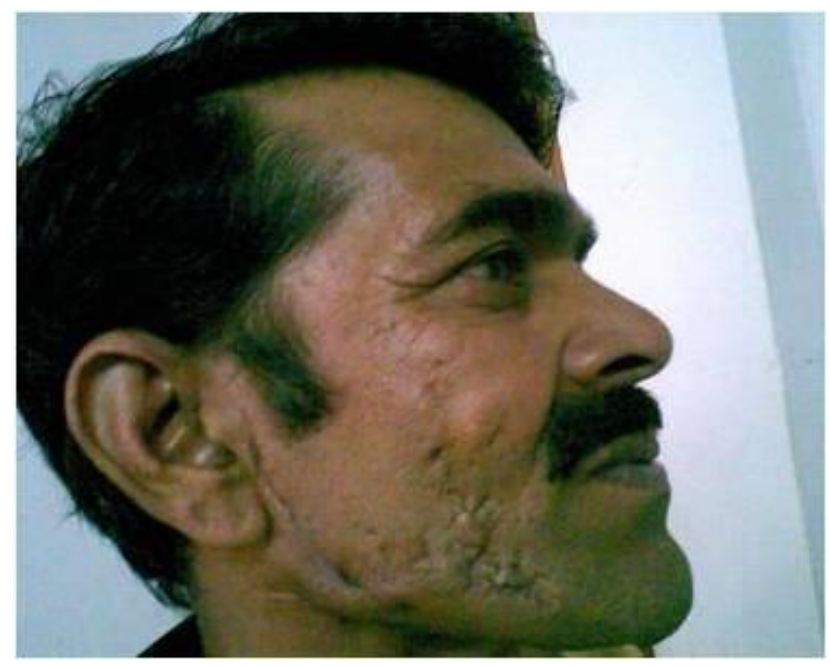

Fig.-2: Appearance of face (disappearing soft tissue nodules), four months after initiation of specific treatment.

\section{Discussion}

Actinomycosis is a benign potentially \& completely curable disease \& not contagious. ${ }^{1}$ The causative bacteria are normally found in mouth, nose, throat \& gastrointestinal tract. But poor hygiene, dental caries, trauma or surgery can increase the risk of infection by these bacteria. It spreads by direct spread to adjacent tissues or by aspiration or through haematogenous route. ${ }^{1}$ In addition to its serious nature of organ involvement, it can complicate other operations or situations like hip prosthesis infection, septic arthritis, endodontic infection, IUD infection, post-operative viscous endophthalmitis. Opportunistic actinomycotic infection has been reported in osteo-radionecrosis in patients having head \& neck cancer. ${ }^{11-18}$ Disseminated actinomycosis by Actinomyces meyeri \& and Actinobacillus actinomycetemcomitans has also been reported. ${ }^{19}$

When infection occurs by these commensal organisms, the infection commonly produces much fibroplastic reaction. When it invades tissues, it causes subacute pyogenic relatively painless inflammation with considerable induration. Systemic symptoms like fever, weight loss are variably present. ${ }^{1}$ One fifth of the cases have primary lesions in the chest \& an equal proportion in the abdomen, most commonly involving the appendix \& caecum. Majority of the lesions occur in cervicofacial region. The lower jaw is more frequently affected. Indurated gum often simulate a bony swelling. Nodules often appears, which then softens 
$\&$ bursts. The overlying indurated skin is bluish in colour. Softening occurs in patches. Abscesses burst through the skin \& sinuses follow. Pulmonary actinomycosis may occur at any age, but most patients are 30-60 years old. Men get this infection more often than women do. ${ }^{5,20}$ Lungs \& pleura are infected either by aspiration or by direct spread from the pharynx or the neck or even upwards through the diaphragm from the abdomen. ${ }^{3}$ The chest wall in the late stages becomes riddled with sinuses. Pulmonary infiltrates, consolidation, hilar manifestations are not uncommon. Perforating the pleural cavity \& the chest wall, the infection may spread to invade the ribs \& vertebra causing osteomyelitis. ${ }^{9}$ The infection can easily spread through the diaphragm to the liver \& the subphrenic space. According to some other authors, abdominal lesions are less common. Lungs, liver, kidney \& skin are sometimes affected by haematogenous route. ${ }^{20}$ It can produce appendicitis, ileocacal mass \& complications thereof. Here surprisingly local \& regional lymph nodes are normal if secondary infection does not supervene.

The sinuses \& fistula tracts may resemble those of furunculosis \& tuberculosis. In tissues, the organism grows in colonies which gradually increase in size \& become visible ${ }^{1}$. These are well-known classical 'sulphur' granules. They are so called because of their yellow colour which ultimately become dark brown or even black. The discharging sinus \& fistula tracts usually become secondarily infected with other bacteria (more commonly with pyogenic cocci).

Laboratory diagnosis of actinomycosis may be done by using specimen like pus, sulphur granulrs, sinus tract, sputum, FNAC \& bsopsy materials. ${ }^{21}$ Microscopy reveals the characteristic 'ray-fungus' (crushed \& stained 'sulphur granules'). Characteristic colonies in solid media \& 'fluff-ball' colonies in liquid media are diagnostic in anaerobic culture. Colonoscopy, CT scans are sometimes required for diagnosis. ${ }^{22}$

Medical treatment includes intensive course of penicillin in large doses for a prolonged period (10 mega units, reducing to 4 mega units daily as long as several months to a year until all signs \& symptoms have disappeared). ${ }^{20}$ The organism is also sensitive to tetracycline \& some other antibiotics like lincomycin, clindamycin \& macrolides (erythromycin, carbomycin, spiramycin, oleandomycin), but the sensitivity should be checked in the laboratory. ${ }^{1,3}$ Recalcitrant actinomycosis sometimes need treatment with ciprofloxacin. ${ }^{23}$ Regular cleaning with normal saline \& application of antibacterial cream like that based on povidone iodine should be applied. Surgery is indicated to take a biopsy specimen, to drain abscesses, to extirpate (wide exicision) a fibrotic sinus tract (not responsive to conservative treatment) or a fistula tract, to decompress or remove a intra-cranial or intraspinal SOLs (space occupying lesions), to remove severely damaged segment of lung or liver \& to repair a defect, or to rule out a bronchogenic carcinoma. ${ }^{24}$

\section{Conclusion}

Diagnosis of Actinomycosis is not difficult. Careful inspection of the discharge for so called 'sulfur granules' preferably under a magnifying glass can give the initial clue to the diagnosis. FNAC \& Biopsy are often helpful, but bacterial identification is essential to confirm the diagnosis. Culture \& Sensitivity tests are sometimes required to have a complete cure of the disease. Timely diagnosis \& initiation of appropriate treatment of Actinomycosis not only cures the patient, but also prevents many complications \& morbidities thereof. Selected cases need surgical intervention.

\section{References}

1. Haldane DJM. Actinomycosis. In: Marrie TJ, ed. Community Acquired Pneumonia. New York: Springer US, 2007:827-840.

2. Weese WC, Smith IM. A study of 57 cases of actinomycosis over a 36-year period. A diagnostic "failure"with good prognosis after treatment. Arch Intern Med 1975;135:1562-8.

3. de Montpreville VT, Nashashibi N, Dulmet EM. Actinomycosis and other bronchopulmonary infections with bacterial granules. Ann Diagn Pathol 1999;3:67-74.

4. Lerner PI. The lumpy jaw:Cervicofacial actinomycosis. Infect Dis Clin North Am 1988;2:203.

5. Jensen BM, Kruse-Anderson S, Anderson K. Thoracic Actinomycosis. Scand J Thoracic Cardiovasc Surg 1989; 23:181.

6. Felekouras E, Menenakos C, Griniatsos J, et al. Liver resection in cases of isolated hepatic actinomycosis: case report and review of the literature. Scand J Infect Dis 2004;36:535-8. 
7. Cintron JR, Del Pino A, Duarte B, et al. Abdominal actinomycosis. Dis Colon Rectum 1996;39:105-8.

8. Huang KL, Beutler SM, Wang C. Endocarditis due to Actinomyces meyeri. Clin Infect Dis 1998;27:90910 .

9. Yung BC, Cheng JC, Chan TT, et al. Aggressive thoracic actinomycosis complicated by vertebral osteomyelitis and epidural abscess leading to spinal cord compression. Spine 2000;25:745-8

10. Rothschild B, Naples V, Barbian L. Bone manifestations of actinomycosis. Ann Diagn Pathol 2006;10:24-27.

11. Lippes J. Pelvic actinomycosis: a review and preliminary look at prevalence. Am J Obstet Gynecol 1999;180(2 Pt 1):265-9.

12. Litwin KA, Jadbabaie F, Villanueva M. Case of pleuropericardial disease caused by Actinomyces odontolyticus that resulted in cardiac tamponade. Clin Infect Dis 1999;29:219-20.

13. Wust J, Steiger U, Vuong H, Zbinden R. Infection of a hip prosthesis by Actinomyces naeslundii. J Clin Microbiol 2000;38:929-30.

14. Lequerre T, Nouvellon M, Kraznowska K, et al. Septic arthritis due to Actinomyces naeslundii: report of a case. Joint Bone Spine 2002;69:499-501.

15. T, Baumgartner JC. Occurrence of Actinomyces in infections of endodontic origin. J Endod 2003;29:54952.
16. Soria-Aledo V, Flores-Pastor B, Carrasco-Prats M, et al. Abdominopelvic actinomycosis: a serious complication in intrauterine device users. Acta Obstet Gynecol Scand 2004;83:863-5.

17. Scarano FJ, Ruddat MS, Robinson A. Actinomyces viscosus postoperative endophthalmitis. Diagn Microbiol Infect Dis 1999;34:115-7.

18. Curi MM, Dib LL, Kowalski LP, et al. Opportunistic actinomycosis in osteoradionecrosis of the jaws in patients affected by head and neck cancer: incidence and clinical significance. Oral Oncol 2000;36:294-9.

19. Kuijper EJ, Wiggerts HO, Jonker GJ, et al. Disseminated actinomycosis due to Actinomyces meyeri and Actinobacillus actinomycetemcomitans. Scand J Infect Dis 1992; 24(5):667-72.

20. Schaal KP, Lee HJ. Actinomycete infections in humans-a review. Gene 1992;115:201-11.

21. Smith AJ, Hall V, Thakker B, Gemmell CG. Antimicrobial susceptibility testing of Actinomyces species with 12 antimicrobial agents. J Antimicrob Chemother 2005;56:407-9.

22. Kim JC, Ahn BY, Kim HC, et al. Efficiency of combined colonoscopy and computed tomography for diagnosis of colonic actinomycosis: a retrospective evaluation of eight consecutive patients. Int J Colorectal Dis 2000;15:236-42.

23. Macfarlane DJ, Tucker LG, Kemp RJ. Treatment of recalcitrant actinomycosis with ciprofloxacin. J Infect 1993;27:177-80.

24. Endo S, Murayama F, Yamaguchi T, et al. Surgical considerations for pulmonary actinomycosis. Ann Thorac Surg 2002;74:185-90. 